\title{
Brain Activity During Cocaine Craving and Gambling Urges: An fMRI Study
}

\author{
Hedy Kober', Cheryl M Lacadie ${ }^{2}$, Bruce E Wexler', Robert T Malison', Rajita Sinha' ${ }^{1,3}$ and \\ Marc N Potenza ${ }^{*, 1,3,4,5}$
}

'Department of Psychiatry, Yale University School of Medicine, New Haven, CT, USA; ${ }^{2}$ Department of Diagnostic Radiology, Yale University School of Medicine, New Haven, CT, USA; ${ }^{3}$ Child Study Center, Yale University School of Medicine, New Haven, CT, USA; ${ }^{4}$ Department of Neurobiology, Yale University School of Medicine, New Haven, CT, USA; ${ }^{5}$ CASAColumbia, Yale University School of Medicine, New Haven, CT, USA

\begin{abstract}
Although craving states are important to both cocaine dependence (CD) and pathological gambling (PG), few studies have directly investigated neurobiological similarities and differences in craving between these disorders. We used functional magnetic resonance imaging ( $\mathrm{fMRI}$ ) to assess brain activity in 103 participants (30 CD, 28 PG, and 45 controls) while they watched videos depicting cocaine, gambling, and sad scenarios to investigate the neural correlates of craving. We observed a three-way urge type $\times$ video type $\times$ diagnostic group interaction in self-reported craving, with CD participants reporting strong cocaine cravings to cocaine videos, and PG participants reporting strong gambling urges to gambling videos. Neuroimaging data revealed a diagnostic group $\times$ video interaction in anterior cingulate cortex/ventromedial prefrontal cortex (mPFC), activating predominantly to cocaine videos in CD participants, and a more dorsal mPFC region that was most strongly activated for cocaine videos in CD participants, gambling videos in PG participants, and sad videos in control participants. Gender $\times$ diagnosis $\times$ video interactions identified dorsal mPFC and a region in posterior insula/caudate in which female but not male PG participants showed increased responses to gambling videos. Findings illustrate both similarities and differences in the neural correlates of drug cravings and gambling urges in CD and PG. Future studies should investigate diagnostic- and gender-specific therapies targeting the neural systems implicated in craving/urge states in addictions.

Neuropsychopharmacology (2016) 4I, 628-637; doi:I0.1038/npp.20I5.193; published online 29 July 2015
\end{abstract}

\section{INTRODUCTION}

Substance-use disorders (SUDs) are chronic relapsing conditions (O'Brien et al, 1998) with substantial societal costs (eg, National Institute of Health, 2008). Cocaine is used by over 40 million Americans and cocaine dependence (CD) is associated with significant health impairments (Sofuoglu and Kosten, 2005). Pathological gambling (PG) and SUDs, including $\mathrm{CD}$, frequently co-occur and share genetic, clinical, and diagnostic features (Leeman and Potenza, 2012). In fact, shared genetic contribution in PG and CD may be more substantial than with other SUDs (Xian et al, 2014). Based on such similarities, PG was recently grouped with SUDs in DSM-5 under 'Substance Related and Addictive Disorders' (APA, 2013; Holden, 2010; Potenza, 2013). Understanding both shared and unique features between PG and SUDs should help advance both prevention and treatment strategies for these disorders and addictions more generally.

Appetitive and motivational states like drug cravings are central to SUDs, including CD (O'Brien et al, 1998; Volkow et al, 2006) and are associated with drug-taking and relapse

*Correspondence: Professor MN Potenza, Department of Psychiatry, Yale University School of Medicine, One Church St. Suite 70I, New Haven, CT 065 I0, USA, E-mail: marc.potenza@yale.edu Received 16 February 2015; revised 14 May 2015; accepted 2 June 2015; accepted article preview online 29 June 2015 (eg, Epstein et al, 2010). Similarly, gambling urges contribute importantly to PG, correlating with problem-gambling severity (Petry et al, 2005) and relapse to gambling (Oei and Gordon, 2008). Consequently, gambling urges have been a target of treatment for PG (Grant et al, 2009).

Given the importance of craving/urges, their neural correlates have been investigated in both CD and PG. For example, Wexler et al (2001) presented CD participants with videos depicting simulated cocaine use. This elicited increased cocaine craving as well as increased activation in medial prefrontal cortex (mPFC), insula, and anterior cingulate cortex (ACC). Similar videos depicting gambling scenarios elicited gambling urges and decreased activation in mPFC and ACC in men with PG (Potenza et al, 2003b). Other studies have observed relatively increased activation in PG male participants to video and picture gambling cues in regions including dorsolateral $\mathrm{PFC}$ and posterior cingulate (Crockford et al, 2005; Goudriaan et al, 2010). We (Potenza, 2008) previously compared the neural correlates of cocaine cravings and gambling urges in men only, and reported similarities between the groups, including relatively diminished activations of ventrolateral PFC during cocaine cravings in men with $\mathrm{CD}$ and gambling urges in men with PG. Differences were also reported, with both dorsal and ventral regions of $\mathrm{mPFC} / \mathrm{ACC}$ relatively more activated during craving in men with $\mathrm{CD}$ compared with $\mathrm{PG}$ 
(Potenza, 2008). However, as these studies have involved small and predominantly or entirely male samples, larger studies involving both men and women are needed to investigate similarities and differences in the neural correlates of gambling urges and cocaine cravings.

It is important to study addictions in a gender-informed fashion (Mitchell and Potenza, 2015), as gender-related differences (We chose to use the term 'gender' based on the recommendations of the Committee for Understanding the Biology of Sex and Gender Differences, established by the Institute of Medicine (Torgrimson and Minson, 2005)) have been reported in both $\mathrm{CD}$ and PG. For example, women progress more rapidly from the initiation of cocaine use or gambling to $\mathrm{CD}$ or PG diagnoses (termed 'telescoping'; Granero et al, 2009; Grant and Kim, 2002; Greenfield et al, 2010; Potenza et al, 2001). Similarly, CD women as compared with men are more likely to consume cocaine via routes that may be more 'addictive,' like smoking (McCance-Katz et al, 1999), and women with PG differ from men in the types of gambling preferred and performed (eg, on electronic-gambling/slot machines; Grant and Kim, 2002; Potenza et al, 2001). In both CD and PG, negative affect contributes more to cocaine use and gambling in women compared with men (Grant and Kim, 2002; McKay et al, 1996). Furthermore, women with CD or PG are more likely to suffer from co-occurring depression and anxiety compared with men with these disorders (Granero et al, 2009; Greenfield et al, 2010; McCance-Katz et al, 1999). Finally, in CD, gender-differences have been reported in response to pharmacological treatments (Kosten et al, 1993; Pettinati et al, 2008) as well as in craving-related brain activations (Kilts et al, 2004; Li et al, 2005; Potenza et al, 2012), although the pattern of differences has been inconsistent. Considering gender-related influences on the neural correlates of craving/urge states may: (1) help resolve and better understand some of the apparent differences observed across studies to date; and (2) provide insight into genderrelated differences in brain function that might relate to important clinical differences observed in PG and CD.

To directly compare neural activity during craving/urge states in women and men with $\mathrm{CD}$ and $\mathrm{PG}$, and control participants (CPs) without either disorder, we recruited 114 participants for functional magnetic resonance imaging (fMRI). Extending our prior work (Balodis et al, 2012b; Kosten et al, 2006; Potenza, 2003b; Wexler et al, 2001), participants viewed three types of videos depicting cocaine, gambling, and sad scenarios. Cocaine and gambling scenarios were designed to induce cravings/urges for cocaine and gambling in CD and PG participants, respectively. Sad videos were included as an emotional (non-urge) active control condition and to examine emotional processing in $\mathrm{CD}$ and PG. As our prior work had identified specific patterns of brain activation during early $v s$ final periods of video viewing (Potenza et al, 2003b; Wexler et al, 2001), we anticipated different patterns of activity during these discrete periods. Furthermore, we hypothesized that activity in regions previously implicated in drug cravings and gambling urges in $\mathrm{CD}$ and $\mathrm{PG}$, including $\mathrm{MPFC}, \mathrm{ACC}$, striatum and insula, would be sensitive to diagnostic group and video type (eg, with CD participants showing greater activation in relation to cocaine videos). We also expected that some of these regions may show additional gender-related differences (ie, gender $\times$ diagnosis $\times$ video type interaction, as has been seen in cravings in CD; Potenza et al, 2012).

\section{MATERIALS AND METHODS}

\section{Participants}

One hundred and fourteen participants (ages 18-59 years, $\mathrm{M}=35.61, \quad \mathrm{SD}=10.89 ; 48$ females) were recruited via advertisements and referrals. Data from this large independent sample have not been previously published. Participants underwent phone screening to determine initial eligibility and were excluded if they were left-handed, did not speak English, were treatment-seeking, or reported pregnancy, claustrophobia, head trauma with loss of consciousness, or any implants or non-removable metal contraindicated in MRI. Eligible participants were administered a structured clinical interview for DSM-IV and the structured clinical interview for PG (SCI-PG; Grant et al, 2004). From the CD group, we excluded individuals with past/present $\mathrm{PG}$, and from the PG group we excluded any individuals with past/ present CD (see Supplementary Table 1 for listing of psychiatric conditions in the sample). From the CP group, we excluded individuals with any active (past 3 month) AxisI disorder. Across groups, participants were excluded if they reported a history of neurological illness, psychiatric hospitalization in the last 6 months (except for CD), or use of psychotropic medications. After complete description of the study, all participants provided informed consent, as approved by the Yale Human Investigation Committee. Eleven participants were dually diagnosed with both past or present CD and PG and were excluded from further analyses. The final sample included 103 participants, of whom 30 were CD (ages 29-51 years, $M=42.93, S D=6.09 ; 12$ female), 28 were $\mathrm{PG}$ (ages $19-59$ years, $\mathrm{M}=33.39, \mathrm{SD}=10.81 ; 8$ female), and 45 CPs were neither CD nor PG (ages $18-54, \mathrm{M}=30.4$, $\mathrm{SD}=10.76 ; 22$ female). The South Oaks Gambling Screen (Lesieur and Blume, 1987) and Fagerstrom Test for Nicotine Dependence (Heatherton et al, 1991), two widely used, valid and reliable measures, were used to assess severity of problem gambling and smoking, respectively. Clinical and demographic characteristics are summarized in Table 1.

\section{In-Scanner Task}

Participants viewed six videos depicting cocaine, gambling, and sad scenarios presented in a counter-balanced order, following our prior work (Kosten et al, 2006; Potenza et al, 2003b; Wexler et al, 2001). In each video, a male or female actor was shown speaking to the viewer and describing a cocaine use, gambling, or sad experience (see Supplementary materials for additional details). Each participant saw two videos from each category, with one male and one female actor. The sequence of each run is described in Figure 1. Each tape was preceded and followed by $30-45 \mathrm{~s}$ of baseline (gray screen). After each run, participants were asked to rate their urge to use cocaine and urge to gamble on a 1-10 scale ( $1=$ not at all, $10=\mathrm{a}$ lot). A mixed ANOVA involving three diagnostic groups (CD, PG, CP) two gender groups $(\mathrm{M}, \mathrm{F})$, three video types (cocaine, gambling, sad), and two urgereport types ('cocaine urge,' 'gambling urge') was used to evaluate participants' in-scanner ratings, with video and urge 
Table I Demographics and Clinical Characteristics of $N=103$

\begin{tabular}{|c|c|c|c|c|c|c|}
\hline & PG-M & PG-F & CD-M & CD-F & CP-M & CP-F \\
\hline Number of subjects & 20 & 8 & 18 & 12 & 23 & 22 \\
\hline \multicolumn{7}{|l|}{ Demographics } \\
\hline Age: years (SD) & $32.70(9.7 I)$ & $35.13(13.77)$ & $43.78(13.06)$ & $41.67(6.46)$ & $31.74(|2.4|)$ & $29.00(8.8 I)$ \\
\hline Race: White & 15 & 5 & 4 & 2 & 15 & 12 \\
\hline \multicolumn{7}{|l|}{ Ethnicity } \\
\hline Hispanic & 4 & 0 & I & 0 & 3 & 2 \\
\hline Non-Hispanic & 16 & 8 & 17 & 12 & 20 & 20 \\
\hline Education: Years (SD) & $13.10(1.29)$ & $13.38(2.50)$ & $13.06(1.14)$ & $12.30(1.06)$ & $14.5(1.85)$ & $15.09(2.54)$ \\
\hline Pathological gambling & 20 & 8 & 0 & 0 & 0 & 0 \\
\hline Daily tobacco user & 10 & 2 & 11 & 9 & 2 & 2 \\
\hline FTND (SD) & $3.12(3.48)$ & $3.33(1.41)$ & $4.91(2.43)$ & $6(3.32)$ & NA & NA \\
\hline SOGS (SD) & $13.53(3.19)$ & $12.86(3.53)$ & $1.2(2.04)$ & $2.11(3.06)$ & $0.45(0.83)$ & $0.53(1.84)$ \\
\hline
\end{tabular}

Abbreviations: CD, cocaine dependent; CP, control participants; FTND, Fagerstrom test of nicotine dependence; F, female; M, male; NA, not available; PG, pathological gambling; SOGS, South Oaks Gambling Screen.

Demographic and clinical characteristics of 103 participants shown by diagnostic group and gender.

Daily tobacco use values were NA for 2 PG-M, I PG-F, 3 CD-M, 3 CD-F, I CP-M, and I CP-F. FTND values were NA for 5 PG-M, 6 PG-F, 7 CD-M, 3 CD-F, and CPs. SOGS values were NA for I PG-M, I PG-F, 3 CD-M, 3 CD-F, 3 CP-M, and 3 CP-F.

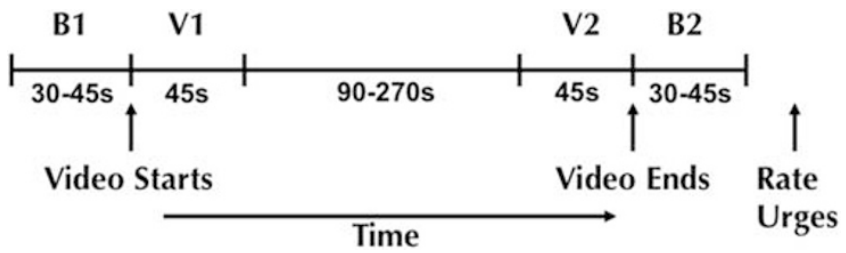

Figure I Schematic representation of each functional run and epochs used in analyses. The epochs in each functional run are schematically represented. $\mathrm{BI}$ is the first baseline period, prior to video viewing (gray screen). VI corresponds to the initial 45 s of video viewing. V2 corresponds to the final $45 \mathrm{~s}$ of video viewing. B2 is the second baseline period, after video viewing (gray screen). The comparisons of interest were $\mathrm{VI}>\mathrm{BI}$, and V2 $>$ B2. Self-reported emotional and motivational ratings were collected after the end of each run.

types as within-subjects factors, and both diagnostic group and gender as between-subjects factors.

\section{Imaging Data Acquisition and Analysis}

Images were obtained using a $3 \mathrm{~T}$ Siemens Trio MRI system. Functional images were acquired in the axial plane parallel to the AC-PC line $\left(\mathrm{TR} / \mathrm{TE}=1500 / 27 \mathrm{~ms}\right.$, flip angle $=60^{\circ}$, field of view $=220 \times 220 \mathrm{~mm}$, and $25 \times 5 \mathrm{~mm}$ slices). In addition, high-resolution 3D Magnetization Prepared Rapid Gradient Echo structural images were acquired for multi-subject registration $\left(\mathrm{TR} / \mathrm{TE}=2530 / 3.34 \mathrm{~ms}\right.$; flip angle $=7^{\circ}$, field of view $=256 \times 256 \mathrm{~mm}$, and $176 \times 1 \mathrm{~mm}$ slices).
The first six images of each run were removed from analysis. Functional images were then slice time-corrected and motion-corrected with SPM5 (Wellcome Functional Imaging Laboratory, UK). Trials with linear motion in excess of $2 \mathrm{~mm}$ or rotation in excess of $3^{\circ}$ were rejected. The corrected images were smoothed with a Gaussian filter with a full-width half maximum of $6.88 \mathrm{~mm}$. Functional images were then registered to each subject's low-resolution and high-resolution structural images, and warped into Montreal-Neurological-Institute space using the Colin27 Brain in Yale BioImage Suite (http://www.bioimagesuite. $\operatorname{org} /$ ). Finally, data were converted to AFNI format (http:// afni.nimh.nih.gov).

Following our prior work (Kosten et al, 2006; Potenza et al, 2003b; Wexler et al, 2001), each functional run was divided into periods of video viewing (Figure 1). The first baseline period (B1), the initial $45 \mathrm{~s}$ of video viewing (V1), the final $45 \mathrm{~s}$ of video viewing (V2), and the last baseline period (B2). For each subject, two comparisons were created: $\mathrm{V} 1>\mathrm{B} 1$ and $\mathrm{V} 2>\mathrm{B} 2$ for each video type (cocaine, gambling, and sad). As reported previously (Kosten et al, 2006; Potenza et al, 2003b; Wexler et al, 2001), the first comparison investigates neural activity associated with the emergence of craving/emotional responses, whereas the second comparison investigates more developed or protracted craving/emotional responses. For each comparison, we performed a random effects $3 \times 2 \times 3$ ANOVA involving three diagnostic groups (CD, PG, CP), two genders $(\mathrm{M}, \mathrm{F})$ and three video types (cocaine, gambling, sad). Results were family-wise error (FWE) corrected for multiple comparisons using Monte Carlo simulation; a voxel-based threshold of.005 with a 56-voxel extent (1538 
Gambling Videos

a

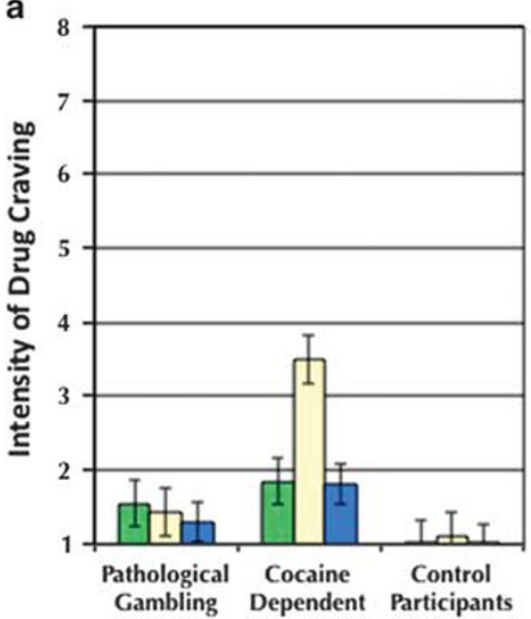

Cocaine Videos $\square$ Sad Videos

b

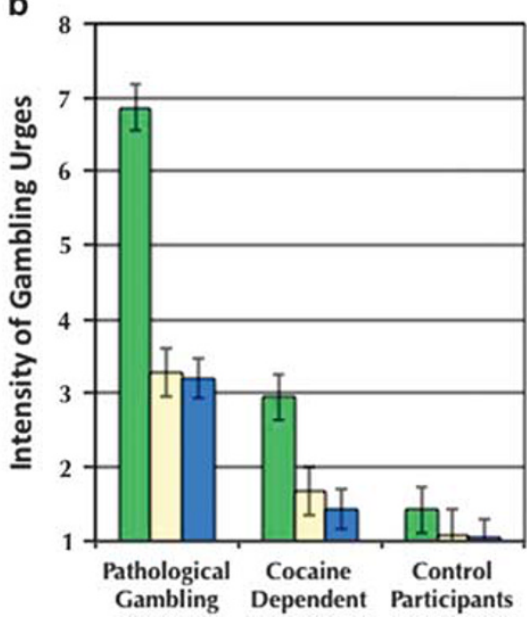

Figure 2 In-scanner subjective ratings. We observed a three-way urge type $\times$ video type $\times$ diagnostic group interaction $\left(F_{(4,194)}=\mid 7.86, P<0.00 I\right)$. It shows, as expected, that (a) cocaine-dependent participants reported strongest cocaine cravings to the cocaine videos and (b) pathological-gambling participants reported strongest gambling urges to the gambling videos.

cubed millimeters) resulted in FWE-corrected $P<0.05$. Results were visualized in BioImage Suite, and percent signal change was extracted from regions that survived whole-brain correction. Post hoc $t$-tests were performed to elucidate the nature of the effects. Covarying for age did not significantly change findings.

\section{RESULTS}

\section{In-Scanner Subjective Ratings}

Full results are presented in the Supplementary materials. Importantly, we observed a three-way urge type $\times$ video type $\times$ diagnostic group interaction on self-reported ratings of urges $\left(\mathrm{F}_{(4,97)}=17.86, P<0.001\right)$, which showed that $\mathrm{CD}$ participants reported highest cocaine urges to cocaine videos and PG participants reported highest gambling urges to gambling videos (Figure $2 \mathrm{a}$ and $\mathrm{b}$, respectively). There were no main or interactive effects involving gender (all $P>0.1$ ).

\section{Imaging Results}

Imaging results are summarized in Table 2.

\section{V1-B1}

A significant main effect of gender was observed in a region that includes the posterior hippocampus and middle temporal gyrus (Supplementary Figure S1). Activity extracted from this region was significantly greater for women than for men $\left(\mathrm{t}_{(101)}=5.21, P<0.001\right)$. A main effect of video type was observed in several regions including bilateral occipital, temporal, and primary motor cortices, hippocampus, and lateral PFC (inferior and middle frontal gyri, bilaterally; Supplementary Figure S2). Signal extracted from each region indicated relatively increased activation for the cocaine videos compared with both gambling and sad videos (all $P<0.001)$.
A diagnostic group $\times$ video type interaction was observed in perigenual ACC/medial PFC (Figure 3a; Supplementary Figure S3). In this region, CD participants showed greater activity for the cocaine videos compared with sad $\left(\mathrm{t}_{(29)}=3.60, P=0.001\right)$ and gambling videos $\left(\mathrm{t}_{(29)}=2.47\right.$, $P<0.05)$. Conversely, CPs exhibited significantly lower activity to cocaine videos compared with gambling $\left(t_{(44)}=2.11\right.$, $P<0.05)$ and sad videos $\left(\mathrm{t}_{(44)}=2.18, P<0.05\right)$. In $P G$ participants activity did not significantly differ between videos. Additional between diagnostic group comparisons showed greater activity in this region for $C D$ and $P G$ relative to $\mathrm{CP}$ groups for cocaine videos $\left(\mathrm{t}_{(73)}=2.92, P=0.005\right.$; $\left.\mathrm{t}_{(71)}=2.21, P<0.05\right)$, and lower activity for CD participants relative to CPs $\left(\mathrm{t}_{(73)}=2.11, P<0.05\right)$ for sad videos.

\section{V2-B2}

During the final period of video viewing, a main effect of gender was observed again in a region that transects the posterior hippocampus and middle temporal gyrus (Supplementary Figure S4), where activity was significantly greater for women than for men $\left(\mathrm{t}_{(64.185)}=4.22, P<0.001\right)$. A main effect of video type was observed in extensive regions of the visual system, encompassing lateral and medial temporal regions (including the amygdalae and hippocampi), extending as far as the anterior insula, bilaterally (Supplementary Figure S5). Activity in this region was greater for gambling videos relative to both sad $\left(\mathrm{t}_{(102)}=6.40, P<0.001\right)$ and cocaine videos $\left(\mathrm{t}_{(102)}=7.91, P<0.001\right)$. Activity was also greater in sad relative to cocaine videos $\left(\mathrm{t}_{(102)}=3.18, P<0.005\right)$. A similar pattern was found in the right middle frontal gyrus/premotor cortex/insula and medial/superior frontal gyrus (SFG) such that activity was greater for gambling compared with both sad and cocaine videos (all $P<0.005$ ). Activity in posterior premotor and motor cortex was greater for sad compared with gambling $\left(\mathrm{t}_{(102)}=2.54, P<0.05\right)$ and cocaine $\left(\mathrm{t}_{(102)}=4.56\right.$, $P<0.001)$ and greater in gambling compared with cocaine videos $\left(\mathrm{t}_{(102)}=2.39, P<0.05\right)$. 
Table 2 Neuroimaging Results: Regions Showing Significant Main or Interactive Effects of Gender, Video Type, and Diagnostic Group

Peak coordinates

\begin{tabular}{|c|c|c|c|c|c|c|c|c|}
\hline \multirow[b]{2}{*}{ Contrast } & \multirow[b]{2}{*}{ Effect/region of activation ${ }^{a}$} & & & & & & & \\
\hline & & $\mathbf{R} / \mathbf{L}$ & $\mathbf{x}$ & $\mathbf{Y}$ & $\mathbf{Z}$ & $\mathbf{K}$ & Vol $\left(\mathrm{mm}^{3}\right)$ & Max Stat \\
\hline \multicolumn{9}{|l|}{$V I>B I$} \\
\hline & Gender & & & & & & & \\
\hline & \multicolumn{8}{|l|}{ Video type } \\
\hline & Visual occipital/middle and superior temporal cortex & $\mathrm{R}$ & 43 & -68 & 4 & 6257 & 168935 & 90.47 \\
\hline & Ventrolateral prefrontal cortex/inferior frontal gyrus & $\mathrm{R}$ & 43 & 30 & 3 & 157 & 4231 & 14.98 \\
\hline & Lateral prefrontal Cortex/inferior and middle frontal gyrus & $\mathrm{L}$ & -49 & 25 & 16 & 155 & 4198 & |4.9| \\
\hline & Middle frontal gyrus/premotor cortex & $\mathrm{L}$ & -49 & -3 & 32 & 127 & 3431 & 16.50 \\
\hline & Middle frontal gyrus/premotor cortex & $\mathrm{R}$ & 37 & -3 & 24 & 205 & 5522 & 20.30 \\
\hline & \multicolumn{8}{|l|}{ Diagnostic group $\times$ Video type } \\
\hline & Hippocampus/medial temporal gyrus & $\mathrm{R}$ & 38 & -48 & 2 & 63 & 1694 & 18.16 \\
\hline & \multicolumn{8}{|l|}{ Video type } \\
\hline & Visual occipital/middle+superior temporal/hippocampus & B & 48 & -25 & 4 & 12355 & 333577 & 101.09 \\
\hline & Premotor cortex/middle frontal gyrus/insula & $\mathrm{R}$ & 46 & 8 & 25 & 119 & 3223 & 12.69 \\
\hline & Superior and medial frontal gyrus & L & -6 & 37 & 41 & 58 & 1563 & 9.62 \\
\hline & Premotor/motor cortex/insula & M & 3 & 10 & 59 & 272 & 7351 & 12.08 \\
\hline & \multicolumn{8}{|l|}{ Diagnostic group $\times$ Video type } \\
\hline & Superior/medial prefrontal/dorsal anterior cingulate cortex & $L$ & -20 & 28 & 17 & 124 & 3358 & 6.40 \\
\hline & \multicolumn{8}{|l|}{ Gender $\times$ Diagnostic group $\times$ Video type } \\
\hline
\end{tabular}

Abbreviations: $K$, number of voxels; L, left; Max stat, F-value at peak voxel; R, right; Vol, volume in cubed millimeters; $X Y Z$, coordinates.

aNote: please see Figures 3, 4 and SI-S7 for visual representations and directionality of effects.

Main and interactive effects of gender (male or female), video type (cocaine, gambling, or sad), and diagnosis (cocaine dependence, pathological gambling, and control participants) in $\mathrm{fMRI}$ data. Results whole-brain family-wise-error corrected at $P<0.05$.

A diagnostic group $\times$ video type interaction was seen in a single region in dorsomedial PFC (dmPFC)/ACC (Figure 3b; Supplementary Figure S6). Activity in this region in PG participants was greatest for the gambling videos, relative to cocaine $\left(\mathrm{t}_{(27)}=281, P<0.01\right)$, and sad videos $\left(\mathrm{t}_{(27)}=1.93\right.$, $P<0.07)$, and also compared with CPs during the gambling video $\left(\mathrm{t}_{(36.08)}=2.33, P<0.01\right)$. In CPs, activity was greatest for sad videos (relative to gambling $\mathrm{t}_{(44)}=3.18, P<0.005$, and cocaine, $\left.\mathrm{t}_{(44)}=1.82, \quad P=0.07\right)$. CD participants showed greater activation during cocaine videos than did PG participants $\left(\mathrm{t}_{(56)}=2.69, P<0.01\right)$, with a marginal difference from CPs $\left(\mathrm{t}_{(73)}=1.81, P=0.07\right)$.

Finally, a three-way gender $\times$ diagnostic group $\times$ video type interaction was observed in putamen, extending to the posterior insula, and in a region including $\mathrm{mPFC} / \mathrm{SFG}$ (Supplementary Figure S7). In the putamen/posterior insula (Figure 4), PG women exhibited greater activity for gambling videos compared with PG men (gambling; $\mathrm{t}_{(26)}=2.42$, $P<0.05)$, and greater activity for gambling $\left(t_{(7)}=3.20\right.$,
$P=0.05)$ and $\operatorname{sad}\left(\mathrm{t}_{(7)}=2.62, P<0.05\right)$ videos compared with cocaine videos. Conversely, activity in this region in $\mathrm{CP}$ and $\mathrm{CD}$ women did not differ between video types (all $P>0.05)$. This pattern was not apparent for men with PG or $\mathrm{CD}$ (all $P \mathrm{~s}>0.1$ ). However, $\mathrm{CP}$ men showed greater activity to gambling videos relative to sad $\left(\mathrm{t}_{(22)}=2.39, P<0.05\right)$ and cocaine $\left(\mathrm{t}_{(22)}=2.92, P<0.01\right)$ videos.

In the $\mathrm{mPFC} / \mathrm{SFG}$ (Figure $4 \mathrm{~b}$ ), $\mathrm{PG}$ men showed greater activity to sad than cocaine videos $\left(\mathrm{t}_{(19)}=2.29, P<0.05\right)$. No other within-group contrasts were significant for men. Similarly, CP women showed greater activation for sad relative to cocaine videos $\left(t_{(21)}=2.73, P<0.02\right)$. CD women showed greater activation for gambling compared with sad $\left(\mathrm{t}_{(11)}=4.27, P<0.005\right.$, respectively) and cocaine $\left(\mathrm{t}_{(11)}=3.07\right.$, $P<0.05)$ videos.

Importantly, significant gender-related differences were evident in this region. PG women showed significantly more activity during cocaine video viewing relative to $P G$ men $\left(\mathrm{t}_{(25.93)}=4.80, P<0.005\right)$. CP women showed greater activity 
a

V1 $>$ B1

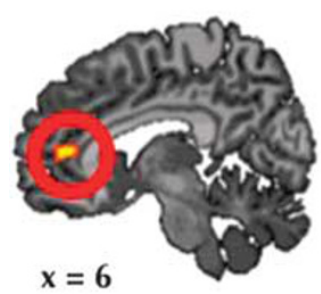

b

$\mathrm{V} 2>\mathrm{B} 2$

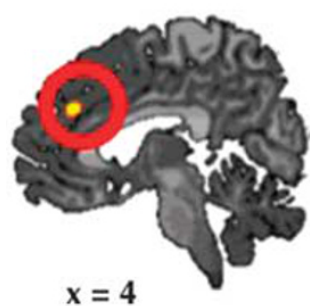

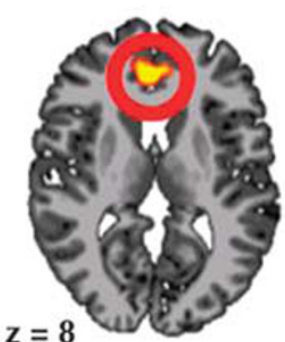
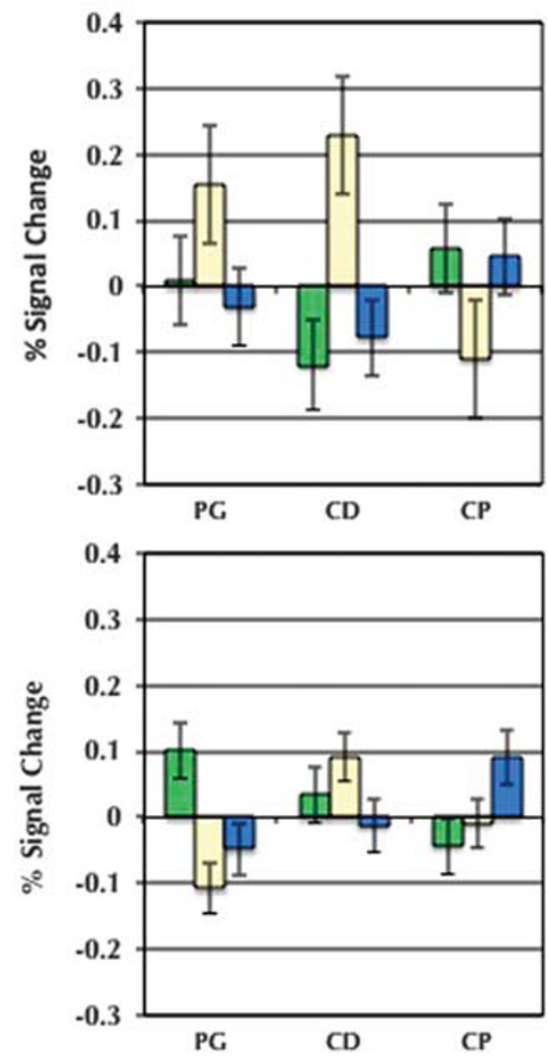

Figure 3 Diagnostic group $\times$ video type interaction. We observed an interaction of diagnostic group $\times$ video type in both contrasts. (a) Activity in the $\mathrm{VI}>\mathrm{BI}$ contrast in a region in the perigenual anterior cingulate cortex that survived whole-brain family-wise-error correction $(P<0.05)$. The graph shows that activity in this region was significantly greater in cocaine-dependent (CD) participants while viewing cocaine videos. (b) Activity in the V2>B2 contrast in a region bordering dorsomedial prefrontal cortex and dorsal anterior cingulate cortex. The graph shows that activity in this region was significantly greater in pathological-gambling (PG) participants while viewing gambling videos, in CD participants while viewing cocaine videos, and in control participants (CP) while viewing sad videos. $\mathrm{BI}$ is the pre-video baseline; VI is the initial period of the video; V2 is the final period of video viewing; $\mathrm{B} 2$ is the post-video baseline.

during the sad video than CP men $\left(\mathrm{t}_{(43)}=2.09, P<0.05\right)$. CD women differed from $\mathrm{CD}$ men for all three video types such that $\mathrm{CD}$ women exhibited greater activity to the gambling video $\left(\mathrm{t}_{(28)}=3.53, P=0.001\right)$ but $\mathrm{CD}$ men showed greater activity to sad $\left(\mathrm{t}_{(28)}=2.84, P<0.01\right)$, and, importantly, cocaine videos $\left(t_{(28)}=2.64, P<0.05\right)$, relative to $C D$ women.

\section{DISCUSSION}

This investigation of the neural correlates of appetitive and motivational (craving/urges) and emotional (sad) processing in PG and CD identified significant main and interactive effects of diagnostic group, video type, and gender. The findings support our a priori hypotheses. Implications are discussed below.

\section{Subjective Responses}

Consistent with our prior work, self-reported data show that relative to CDs and CPs, PG participants reported increased gambling urges to gambling videos, and that relative to PGs and CPs, CD participants reported greater cocaine craving to cocaine videos. This indicates that the videos increased urges to use drugs or gamble in content- and diagnostic group- specific manners. However, gender-related main or interactive effects in self-reported cravings or urges were not observed, consistent with prior reports in CD (Li et al, 2005; Potenza et al, 2012). Indeed, despite a common stereotype that women may be more 'emotional' relative to men (eg, Timmers et al, 2003), similarities in self-reported emotion are common (see Barrett and Bliss-Moreau, 2009 for review).

\section{Imaging Results}

In the first viewing period we observed a video type $\times$ diagnostic group interaction in perigenual ACC/mPFC, where $C D$ participants showed increased activity while watching cocaine videos compared with both PG participants and CPs. This region has been previously associated with cocaine craving in $\mathrm{CD}$, thus replicating and extending prior findings in predominantly/solely male CD samples (Potenza, 2008; Wexler et al, 2001). Conversely, some studies with PG have reported hypoactivations in vmPFC during gambling urges (eg, Potenza et al, 2003b, but cf, Goudriaan et al, 2010). Reduced activity in this region has also been reported in PG during cognitive control and monetary tasks (Balodis et al, 2012a; Potenza et al, 2003a).

Across video types and diagnostic groups, women as compared with men exhibited greater activity in the 

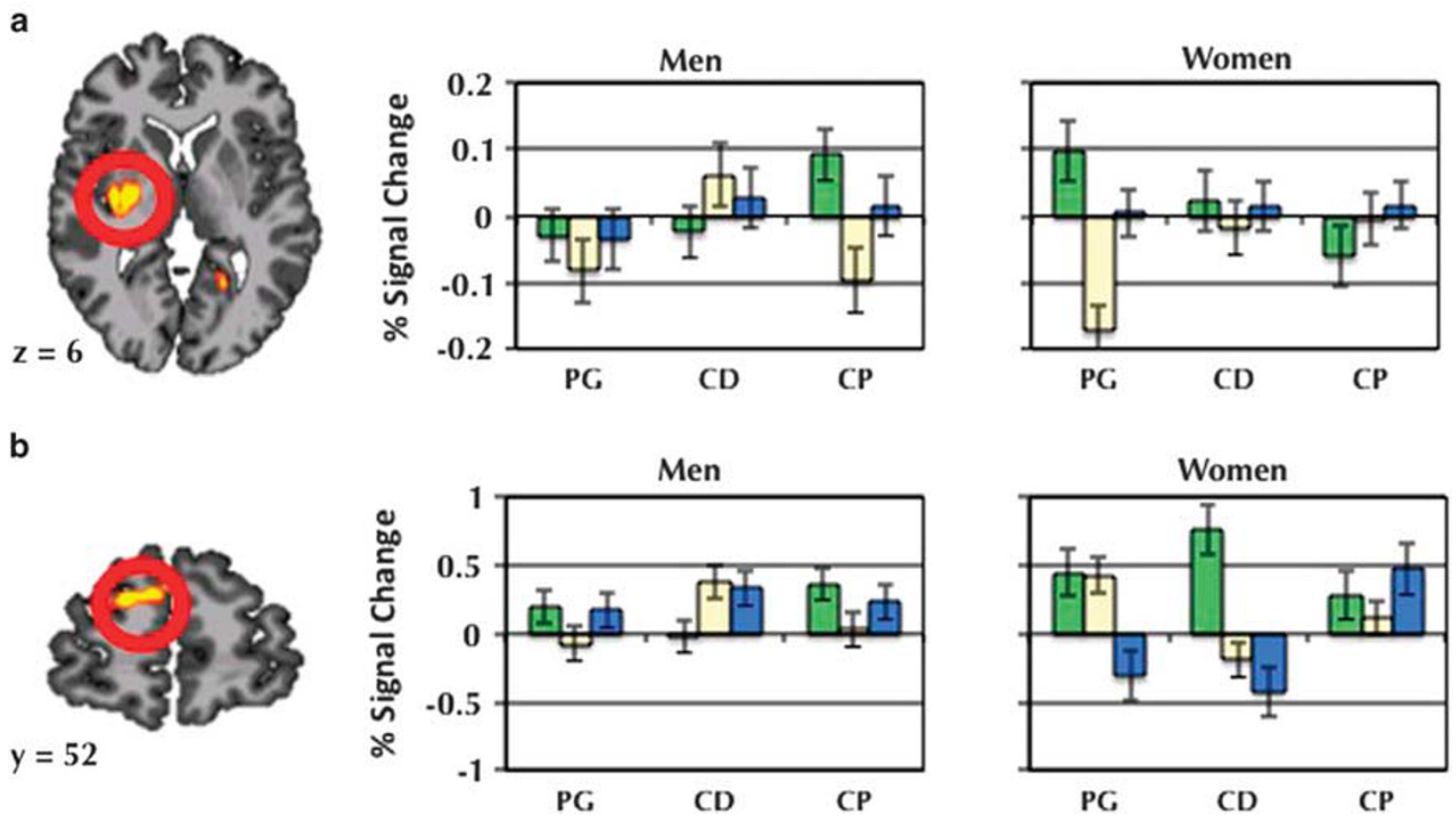

Figure 4 Gender $\times$ diagnostic group $\times$ video type interaction in the V2>B2 contrast. We observed three-way gender $\times$ diagnostic group $\times$ video type interactions during the V2>B2 contrast. (a) A region that includes posterior portions of putamen and insula is shown. Graphs show extracted percent signal change for men (left) and women (right). PG women exhibited greater activity to gambling videos compared with PG men. (b) A region of superior frontal gyrus is shown. Graphs show extracted percent signal change for men (left) and women (right). CD men exhibited greater activity to both cocaine and sad movies compared with CD women. Displayed results were identified using a voxel-wise threshold of $P<0.005$ and survived whole-brain family-wise-error correction $(P<0.05)$. V2 is the final period of the video viewing; B2 is the post-video baseline.

hippocampus and middle temporal gyrus. Given the affective content in the videos and the role of these regions in memory (Eichenbaum, 2004), it may be that memory retrieval is involved in the generation of craving/urge-related emotions and motivations (Balodis et al, 2012b; Kober et al, 2008) and that women activate these regions more so than men during this process. Memory-related activity would be consistent with theories that view the videos as eliciting motivational responses, which are related to participants' 'current concerns' about drug use or gambling (eg, Klinger and Cox, 2004).

Finally, video-related activity across extensive occipital and temporal visual regions, as well as hippocampal, premotor, and frontal regions, was greatest for cocaine videos. This may reflect some visual differences between videos in that the cocaine videos depicted more activity in the first period of video viewing (preparing to use cocaine, compared with talking about a sad event or about opportunities to gamble).

In the last period of video viewing, we observed a video type $\times$ diagnosis interaction in a region of $\mathrm{dmPFC/dACC}$. Activity was selectively increased in a video-specific and diagnosis-specific fashion: gambling videos in PG, cocaine videos in $\mathrm{CD}$, and sad videos in $\mathrm{CP}$ groups. The 'matched' responding in this region suggests that activity underlies the motivational/emotional response generated for these videos, and is consistent with several roles proposed for this region including in emotion (Kober et al, 2008), emotion regulation (Ochsner and Gross, 2005), attention (Weissman et al, 2005), reward-based decision making (Bush et al, 2002), and mental state attribution (Mitchell et al, 2005). We also found a threeway gender $\times$ diagnosis $\times$ video type interactions in regions of putamen/posterior insula and $\mathrm{dmPFC/SFG}$. In the region encompassing the posterior putamen and insula, PG women (but not men) exhibited increased activity for gambling videos. The insula is a large and heterogeneous region with multiple functions, including a general role in interoception, with the posterior insula particularly involved in integrating sensory inputs (Craig, 2009). In addition, it has been suggested that it is directly involved in response to drug cues (Childress et al, 2008; Yalachkov et al, 2012), drug craving (Garavan, 2010), and gambling urges (Goudriaan et al, 2010). Further, damage to this region has been linked to rapid smoking cessation and the elimination of cigarette craving (Naqvi et al, 2007) and abolishes cognitive distortions that drive gambling behavior (eg, 'gambler's fallacy'; Clark et al, 2014). Similarly, the putamen has a role in coding both action and associated reward value (Hori et al, 2009), and is also often activated by drug cues during drug craving (McClernon et al, 2008). Taken together, this finding suggests that PG women may have a unique sensory and motivational response in these regions relative to PG men and non-PG women when encountering gambling-related stimuli. In dmPFC/SFG, gender-related differences were particularly salient for the $\mathrm{CD}$ group: $\mathrm{CD}$ men exhibited greater activity for cocaine and sad videos compared with $\mathrm{CD}$ women, who exhibited greater activity to the gambling videos. 
We also observed significant gender-related differences among CD participants, whereby men but not women with $\mathrm{CD}$ activated this region strongly to cocaine videos. Given the aforementioned role of the dmPFC in emotion and emotion regulation, this pattern may suggest that women in both patient groups differ from $\mathrm{CPs}$ in processing the emotional content of sad material. In addition, these genderrelated differences specifically within $\mathrm{CD}$ participants suggest that $\mathrm{CD}$ is associated with gender-specific alterations in the processing of emotional stimuli, and cocaine-related stimuli in particular. We also found a main effect of gender in hippocampal and medial temporal regions, similar to the first period of video viewing. Finally, we found a main effect of video type in bilateral occipital and temporal visual processing regions, as well as prefrontal regions, in which activity was greatest for the gambling videos. Similar to the first viewing period, this pattern may reflect the visual properties of the videos, as more gambling-related activity was depicted in this period in the gambling compared with the cocaine or sad videos.

These findings have several important clinical implications. As reviewed previously, craving is a key feature of both SUDs (O'Brien et al, 1998; Volkow et al, 2006) and PG (Potenza et al, 2003b) that predicts drug use, gambling, and relapse to those behaviors (eg, Epstein et al, 2010). Consistently, commonly practiced and effective treatments for these disorders include components on identifying triggers for craving and on regulation of craving (such as relapse prevention and cognitive-behavioral therapy; Carroll, 2008; Grant et al, 2009). Furthermore, the regulation strategies used in such treatments have been shown to directly reduce craving (Kober et al, 2010a) as well as neural activity in regions that instantiate craving (Kober et al, 2010b; Volkow et al, 2010). The current work further suggests that craving states may be instantiated differently across genders, indicating that treatments may be made more effective by elucidating gender-specific interventions that target craving.

Limitations of this work include the long and variable duration of the videos used, which was addressed by selectively comparing the first and last video viewing period, following prior work with these or similar videos (Potenza et al, 2003b; Wexler et al, 2001). The lack of non-emotional control videos is another limitation of this study. Additional limitations include the non-equal sample sizes of men, women, and cigarette smokers between groups; however, this reflects typical clinical characteristics of the samples studied (Desai and Potenza, 2008). The exclusion of participants with comorbid PG and CD also limits the generalizability of findings to individuals with either PG or CD diagnoses. A strength of the study involves the use of an established task in a large sample that permitted the identification of main and interactive influences on regional activation related to gender, diagnosis, and video type, with whole-brain-corrected thresholds.

Taken together, these findings from a large sample of 103 participants illustrate both diagnostic group and genderrelated patterns of neural responding associated with drug craving and gambling urges in $\mathrm{CD}$ and $\mathrm{PG}$, respectively. Similarities in neural correlates of craving/urges across diagnostic groups suggest possible targets for treatments across $\mathrm{CD}$ and PG. The findings replicate prior work showing that activity in ACC/mPFC may underlie cocaine cravings in $\mathrm{CD}$, and extend prior work by demonstrating this effect across genders. Unique to this work are the observed gender-, diagnosis-, and video-related differences in neural responses in $\mathrm{mPFC}$, putamen, and posterior insula, all regions previously implicated in craving and affect processing. Although the exact role of these regions remains to be more precisely elucidated, the current findings highlight the need to systematically examine gender-related differences to understand the neural substrates of both disorders. Such research is important to increase our understanding of both substance and non-substance addictions. Future work could specifically address whether the differences we observed may be related to altered emotional, physiological, or motivational responses within each gender group and across disorders, and how these findings might be used to generate improved prevention and treatment strategies for women and men.

\section{FUNDING AND DISCLOSURE}

The Bioimagesuite software used in the fMRI analysis was created with support from the National Institute of Biomedical Imaging and Bioengineering (NIBIB) under grant R01 EB006494. This work was supported by National Institute of Drug Abuse (NIDA) grants K12-DA00167, R01DA019039, P50-DA016556, K24-DA017899, the VA VISN1 MIRECC, a Center of Research Excellence Award from the National Center for Responsible Gaming and its affiliated Institute for Research on Gambling Disorders, as well as the State of Connecticut Department of Mental Health and Addiction Services (DMHAS). This publication was made possible by the CTSA Grant UL1 RR024139 from the National Center for Research Resources (NCRR), a component of the National Institutes of Health (NIH), and NIH roadmap for Medical Research. The contents of the manuscript are solely the responsibility of the authors and do not necessarily represent the official views of any of the funding agencies. Dr Wexler has equity in and receives compensation from C8 Sciences, has equity in Becaid Educational Technology Company, and is a paid consultant for Peak. All three develop computer-presented brain training programs.' The authors report that they have no financial conflicts of interest with respect to the content of this manuscript. Dr Potenza has received financial support or compensation for the following: has consulted for Boehringer Ingelheim, Somaxon, Lundbeck, Ironwood, INSYS, Shire, and RiverMend Health; has received research support from the National Institutes of Health, Veteran's Administration, Mohegan Sun Casino, the National Center for Responsible Gaming, and Forest Laboratories, OrthoMcNeil, Oy-Control/Biotie, Glaxo-SmithKline, Psyadon, and Pfizer pharmaceuticals; has participated in surveys, mailings, or telephone consultations related to drug addiction, impulse-control disorders or other health topics; has consulted for law offices and the federal public defender's office in issues related to impulse-control disorders; provides clinical care in the Connecticut Department of Mental Health and Addiction Services Problem Gambling Services Program; has performed grant reviews for the National Institutes of Health and other agencies; has guest-edited journal sections; has given academic lectures in grand rounds, CME events and other clinical or scientific venues; and has generated books or book chapters for publishers of mental health texts. 


\section{ACKNOWLEDGMENTS}

The authors would like to thank Jennifer Bellegarde, Michael Bernabeo, Scott Bullock, Jessica Montoya, Monica Solorzano, Jocelyn Topf, Katherine VanBuskirk, Mary Wilber, Patrick Worhunsky, and Kourosh Zakeri for help with scanning; Iris Balodis, Scott Bullock, Cameron DeLeone, Maggie Mae Mell, Matthew Schafer, and Kourosh Zakeri for help with preparation of the manuscript; and Xoli J Redmond and Bruce J Rounsaville for their support during the preparation of this manuscript.

\section{REFERENCES}

APA (2013). Diagnostic and Statistical Manual of Mental Disorders. 5th edn. American Psychiatric Publishing: Arlington, VA, USA.

Balodis IM, Kober H, Worhunsky PD, Stevens MC, Pearlson GD, Potenza MN (2012a). Diminished frontostriatal activity during processing of monetary rewards and losses in pathological gambling. Biol Psychiatry 71: 749-757.

Balodis IM, Lacadie CM, Potenza MN (2012b). A preliminary study of the neural correlates of the intensities of self-reported gambling urges and emotions in men with pathological gambling. J Gambl Stud 28: 493-513.

Barrett LF, Bliss-Moreau E (2009). She's emotional. He's having a bad day: attributional explanations for emotion stereotypes. Emotion 9: 649-658.

Bush G, Vogt BA, Holmes J, Dale AM, Greve D, Jenike MA et al (2002). Dorsal anterior cingulate cortex: a role in reward-based decision making. Proc Natl Acad Sci USA 99: 523-528.

Carroll KM (2008). Cognitive-behavioral therapies. In: Galanter M, Kleber HD (eds). The American Psychiatric Publishing Textbook of Substance Abuse Treatment. American Psychiatric Publishing: Arlington, VA, USA, 4th edn, pp 349-360.

Childress AR, Ehrman RN, Wang Z, Li Y, Sciortino N, Hakun J et al (2008). Prelude to passion: limbic activation by 'unseen' drug and sexual cues. PLoS One 3: 1-7.

Clark L, Studer B, Bruss J, Tranel D, Bechara A (2014). Damage to insula abolishes cognitive distortions during simulated gambling. Proc Natl Acad Sci USA 111: 6098-6103.

Craig A (2009). How do you feel-now? The anterior insula and human awareness. Nat Rev Neurosci 10: 59.

Crockford D, Goodyear B, Edwards J, Quickfall J, el-Guebaly N (2005). Cue-induced brain activity in pathological gamblers. Biol Psychiatry 58: 787-795.

Desai RA, Potenza MN (2008). Gender differences in the associations between past-year gambling problems and psychiatric disorders. Soc Psychiatry Psychiatr Epidemiol 43: 173-183.

Eichenbaum H (2004). Hippocampus: cognitive processes and neural representations that underlie declarative memory. Neuron 44: 109-120.

Epstein DH, Marrone GF, Heishman SJ, Schmittner J, Preston KL (2010). Tobacco, cocaine, and heroin: craving and use during daily life. Addict Behav 35: 318-324.

Garavan H (2010). Insula and drug cravings. Brain Struct Funct 214: 593-601.

Goudriaan AE, De Ruiter MB, Van Den Brink W, Oosterlaan J, Veltman DJ (2010). Brain activation patterns associated with cue reactivity and craving in abstinent problem gamblers, heavy smokers and healthy controls: an fMRI study. Addict Biol 15: 491-503.

Granero R, Penelo E, Martínez-Giménez R, Álvarez-Moya E, Gómez-Peña M, Aymami M et al (2009). Sex differences among treatment-seeking adult pathologic gamblers. Compr Psychiatry 50: 173-180.
Grant J, Donahue C, Odlaug B, Kim S, Miller M, Petry N (2009). Imaginal desensitisation plus motivational interviewing for pathological gambling: randomised controlled trial. B J Psychiatry 195: 266-267.

Grant J, Kim S (2002). Gender differences in pathological gamblers seeking medication treatment. Compr Psychiatry 43: 56-62.

Grant JE, Steinberg MA, Kim SW, Rounsaville BJ, Potenza MN (2004). Preliminary validity and reliability testing of a structured clinical interview for pathological gambling. Psychiatry Res 128: 79-88.

Greenfield S, Back S, Lawson K, Brady K (2010). Substance abuse in women. Psychiatr Clin North Am 33: 339-355.

Heatherton TF, Kozlowski LT, Frecker RC, Fagerstrom KO (1991). The fagerstrom test for nicotine dependence: a revision of the fagerstrom tolerance questionnaire. Addiction 86: 1119-1127.

Holden C (2010). Behavioral addictions debut in proposed DSM-V. Science 327: 935-935.

Hori Y, Minamimoto T, Kimura M (2009). Neuronal encoding of reward value and direction of actions in the primate putamen. J Neurophysiol 102: 3530.

Kilts CD, Gross RE, Ely TD, Drexler KPG (2004). The neural correlates of cueinduced craving in cocaine-dependent women. Am J Psychiatry 161: 233-241.

Klinger E, Cox WM (2004). Motivation and the theory of current concerns. In: Cox WM, Klinger E (eds), Handbook of Motivational Counseling: Motivating People for Change. Wiley: Chichester, England, pp 3-23.

Kober H, Barrett LF, Joseph J, Bliss-Moreau E, Lindquist K, Wager TD (2008). Functional grouping and cortical-subcortical interactions in emotion: a meta-analysis of neuroimaging studies. Neuroimage 42: 998-1031.

Kober H, Kross EF, Mischel W, Hart CL, Ochsner KN (2010a). Regulation of craving by cognitive strategies in cigarette smokers. Drug Alcohol Depend 106: 52-55.

Kober H, Mende-Siedlecki P, Kross EF, Weber J, Mischel W, Hart CL et al (2010b). Prefrontal-striatal pathway underlies cognitive regulation of craving. Proc Nat Acad Sci USA 107: 14811-14816.

Kosten TA, Gawin FH, Kosten TR, Rounsaville BJ (1993). Gender differences in cocaine use and treatment response. J Subs Abuse Trea 10: 63-66.

Kosten TR, Scanley BE, Tucker KA, Oliveto A, Prince C, Sinha R et al (2006). CueInduced brain activity changes and relapse in cocainedependent patients. Neuropsychopharmacology 31: 644-650.

Leeman RF, Potenza MN (2012). Similarities and differences between pathological gambling and substance use disorders: a focus on impulsivity and compulsivity. Psychopharmacology 219: $1-22$.

Lesieur HR, Blume SB (1987). The South Oaks Gambling Screen (SOGS): a new instrument for the identification of pathological gamblers. Am J Psychiatry 144: 9.

Li CSR, Kosten TR, Sinha R (2005). Sex differences in brain activation during stress imagery in abstinent cocaine users: a functional magnetic resonance imaging study. Biol Psychiatry 57: 487-494.

McCance-Katz E, Carroll K, Rounsaville B (1999). Gender differences in treatmentseeking cocaine abusers-implications for treatment and prognosis. Am J Addict 8: 300-311.

McClernon FJ, Kozink RV, Lutz AM, Rose JE (2008). 24- h smoking abstinence potentiates fMRI-BOLD activation to smoking cues in cerebral cortex and dorsal striatum. Psychopharmacology 204: 25-35.

McKay J, Rutherford M, Cacciola J, Kabasakalian-McKay R, Alterman A (1996). Gender differences in the relapse experiences of cocaine patients. J Nerv Ment Dis 184: 616-622.

Mitchell J, Banaji M, MacRae C (2005). The link between social cognition and selfreferential thought in the medial prefrontal cortex. J Cogn Neurosci 17: 1306-1315.

Mitchell MR, Potenza MN (2015). Importance of sex differences in impulse control and addictions. Front Psychiatry 6: 24. 
Naqvi NH, Rudrauf D, Damasio H, Bechara A (2007). Damage to the insula disrupts addiction to cigarette smoking. Science 315: 531-534.

National Institutes of Health (2008). Understanding drug abuse and addiction. In: National Institute of Drug Abuse - NIDA InfoFacts, pp 1-4.

O'Brien CP, Childress AR, Ehrman R, Robbins SJ (1998). Conditioning factors in drug abuse: can they explain compulsion? J Psychopharmacology 12: 15-22.

Ochsner KN, Gross JJ (2005). The cognitive control of emotion. Trends Cogn Sci 9: 242-249.

Oei T, Gordon L (2008). Psychosocial factors related to gambling abstinence and relapse in members of gamblers anonymous. J Gambl Stud 24: 91-105.

Petry N, Stinson F, Grant B (2005). Comorbidity of DSM-IV pathological gambling and other psychiatric disorders: results from the National Epidemiologic Survey on Alcohol and Related Conditions. J Clin Psychiatry 66: 564-574.

Pettinati H, Kampman K, Lynch K, Suh J, Dackis C, Oslin D et al (2008). Gender differences with high-dose naltrexone in patients with co-occurring cocaine and alcohol dependence. J Subst Abuse Treat 34: 378-390.

Potenza MN (2008). The neurobiology of pathological gambling and drug addiction: an overview and new findings. Philos Trans $R$ Soc Biol Sci 363: 3181-3189.

Potenza MN (2013). Neurobiology of gambling behaviors. Curr Opin Neurobiol 18: 429-438.

Potenza MN, Hong KA, Lacadie CM, Fulbright RK, Bergquist KL, Sinha R (2012). Neural correlates of stress-induced and cueinduced craving: influences of gender and cocaine dependence. Am J Psychiatry 169: 406-414.

Potenza MN, Leung H-C, Blumberg HP, Peterson BS, Fulbright RK, Lacadie CM et al (2003a). An FMRI stroop task study of ventromedial prefrontal cortical function in pathological gamblers. Am J Psychiatry 2003; 160: 1990-1994.
Potenza MN, Steinberg M, McLaughlin S, Wu R, Rounsaville B, O'Malley S (2001). Gender-related differences in the characteristics of problem gamblers using a gambling helpline. Am J Psychiatry 158: 1500-1505.

Potenza MN, Steinberg MA, Skudlarski P, Fulbright RK, Lacadie CM, Wilber MK et al (2003b). Gambling urges in pathological gambling: a functional magnetic resonance imaging study. Arch Gen Psychiatry 60: 828-836.

Sofuoglu M, Kosten TR (2005). Novel approaches to the treatment of cocaine addiction. CNS Drugs 19: 13-25.

Timmers M, Fischer A, Manstead A (2003). Ability versus vulnerability: beliefs about men's and women's emotional behaviour. Cogn Emot 17: 41-63.

Torgrimson BN, Minson CT (2005). Sex and gender: what is the difference? J Appl Physiol 99: 785-787.

Volkow ND, Fowler JS, Wang GJ, Telang F, Logan J, Jayne M et al (2010). Cognitive control of drug craving inhibits brain reward regions in cocaine abusers. Neuroimage 49: 2536-2543.

Volkow ND, Wang GJ, Telang F, Fowler JS, Logan J, Childress AR et al (2006). Cocaine cues and dopamine in dorsal striatum: mechanism of craving in cocaine addiction. J Neurosci 26: 6583-6588.

Weissman DH, Gopalakrishnan A, Hazlett CJ, Woldorff MG (2005). Dorsal anterior cingulate cortex resolves conflict from distracting stimuli by boosting attention toward relevant events. Cereb Cortex 15: 229-237.

Wexler BE, Gottschalk CH, Fulbright RK, Prohovnik I, Lacadie CM, Rounsaville BJ et al (2001). Functional magnetic resonance imaging of cocaine craving. Am J Psychiatry 158: 86-95.

Xian H, Giddens JL, Scherrer JF, Eisen SA, Potenza MN (2014). Environmental factors selectively impact co-occurrence of problem/pathological gambling with specific druguse disorders in male twins. Addiction 109: 635-644.

Yalachkov Y, Kaiser J, Naumer MJ (2012). Functional neuroimaging studies in addiction: multisensory drug stimuli and neural cue reactivity. Neuroscience Biobehav Rev 36: 825-835.

Supplementary Information accompanies the paper on the Neuropsychopharmacology website (http://www.nature.com/npp) 